\title{
An 8-week randomized controlled trial on the effects of brisk walking, and brisk walking with abdominal electrical muscle stimulation on anthropometric, body composition, and self-perception measures in sedentary adult women
}

\author{
Ailsa G. Anderson ${ }^{\mathrm{a}, *}$, Marie H. Murphy ${ }^{\mathrm{b}}$, Elaine Murtagh ${ }^{\mathrm{b}}$, Alan Nevill ${ }^{\mathrm{c}}$ \\ ${ }^{a}$ School of Life Sciences, Heriot Watt University, Riccarton, Edinburgh, Scotland, EH14 4AS, UK \\ ${ }^{\mathrm{b}}$ University of Ulster, UK \\ ${ }^{\mathrm{c}}$ University of Wolverhampton, $U K$
}

Received 2 August 2005; received in revised form 26 April 2006; accepted 28 April 2006

Available online 15 June 2006

\begin{abstract}
Objectives: The aim of this randomized controlled trial was to examine the effects of an 8-week program of regular brisk walking, regular brisk walking with abdominal electrical muscle stimulation (EMS), and no exercise on hierarchical self-perceptions, and consider the mediating role of changes in anthropometric measures and body composition.

Methods: Thirty-seven sedentary healthy women (mean age $=38.1 ; \mathrm{SD}=9.3$ ) provided written informed consent and participated in baseline testing on a range of anthropometric, body composition, and hierarchical self-perception measures. Subsequently participants were randomly assigned to an 8-week program of walking $(n=13)$, walking + EMS $(n=14)$, or a control $(n=10)$ condition. At 8 weeks anthropometric, body composition and self-perception measures were re-assessed.

Results: In comparison with the control group, both walking groups had significant reductions in a number of anthropometric measures and improvements in self-perception measures. The improvements on both anthropometric measures and self-perceptions were greater for the walking + EMS condition, which indicated that changes in self-perception might be mediated by body changes. However, an assessment of the mediation effect between changes in anthropometric measures and self-perception changes did not support this finding.
\end{abstract}

\footnotetext{
${ }^{*}$ Corresponding author. Tel./fax: + 441314513315.

E-mail address: a.anderson@hw.ac.uk (A.G. Anderson).
} 
Conclusions: An 8-week exercise program results in significant improvements in anthropometric measures and self-perceptions compared with no exercise. Changes in anthropometric measures appear to have limited influence on exercise-induced changes in self-perception and it is suggested that a subjective feeling that one's body is improving may be sufficient to enhance self-perceptions.

(C) 2006 Elsevier Ltd. All rights reserved.

Keywords: Self-perceptions; Physical activity; Brisk walking

\section{Introduction}

Self-esteem and physical self-worth contribute significantly to overall psychological well-being and quality of life, and research indicates that physical activity can positively influence these selfperceptions (Biddle \& Mutrie, 2001; Fox, 2000). Drawing from current multi-dimensional hierarchical theories of self-esteem, Sonstroem and colleagues proposed the Exercise and SelfEsteem Model (EXSEM) and suggested that a positive experience of exercise could enhance exercise self-efficacy, which in turn may benefit one's physical self-perceptions and ultimately lead to changes in self-esteem (Sonstroem, Harlow, \& Josephs, 1994; Sonstroem \& Morgan, 1989).

Fox (2000) reported that out of 36 randomized controlled studies examined, $78 \%$ indicated that exercise led to positive changes in some aspect of physical self-esteem or concept, and argued that this was a robust and significant finding. More recent experimental studies have, to an extent, supported this. For example, Taylor and Fox (2005) reported that participants in a 10-week primary care exercise referral intervention had greater improvements in self-perceptions at 16 and 37 weeks than a control group. Asçi $(2002,2003)$ also found that compared with non-active control groups, participants in 10-week step and aerobic dance programs significantly improved on aspects of physical concept. Further, Alfermann and Stoll (2000) conducted two field experiments with middle-aged adults to examine the effects of exercise on self-concept and wellbeing. The results of the first study indicated that a 6-month exercise program lead to significant changes in physical self-concept compared with a waiting list control group. However, Alfermann and Stoll noted that these changes could be due to expectancy or attentional effects and therefore conducted a second study that compared changes in self-concept over 6 months between two exercising groups and two placebo attention groups. The results of the second study demonstrated that all groups significantly improved indicating that exercise is one, but not the only strategy to improve self-perceptions. Finally, some studies have failed to report an improvement in selfperceptions following exercise (e.g., Asçi, Kin, \& Kosar, 1998; Caruso \& Gill, 1992).

In line with Sonstroem et al.'s (1994) hierarchical model, the relationship between exercise and self-perceptions appears to be stronger and more consistent for the lower-level physical selfperceptions than the more stable construct of global self-esteem, which is at the apex of the hierarchy (Alfermann \& Stoll, 2000; Fox, 2000). However, there has been limited experimental research using multi-dimensional measures to examine the effects of exercise on the hierarchy of self-perceptions (McAuley, Blissmer, Katula, Duncan, \& Mihalko, 2000; Sonstroem, 1998).

The reasons why a positive relationship exists between exercise and self-perceptions are not fully understood. As indicated above, some researchers have suggested that this improvement may be due merely to an expectancy effect that participating in exercise will improve well-being 
(Desharnais, Jobin, Cote, Levesque, \& Godin, 1993). Further, it may be that it is not exercise per se that leads to improvements in self-perception, as other interventions appear to be equally effective (Alfermann \& Stoll, 2000). Fox (2000) suggested that there are likely to be several mechanisms operating such as increased personal autonomy, increased sense of belongingness, and increased perceptions of competence from exercise leading to enhanced self-perceptions. A further possible explanation, which is the focus of this investigation, is that exercise leads to weight loss and improved muscle tone and body size that, in turn, enhances self-perceptions such as body image.

Body image incorporates our thoughts about the body and includes body dissatisfaction (Bane \& McAuley, 1998). In women, body dissatisfaction is positively related to body mass, body fat content, and specific anatomical features such as size of hips, buttocks, and stomach (Bailey, Goldbergh, Swap, Chomitz, \& Houser, 1990; Davis, 1997). Therefore, exercise-induced improvements to the body may result in enhanced body image. Fox (1998) indicated that situation-specific self-perceptions such as feeling fat in certain clothes could be changed through exercise. Consistent with Sonstroem et al.'s (1994) EXSEM model, Fox suggested that this situation-specific change could then influence general feelings of fatness that could subsequently lead to enhanced perceptions of body image and body attractiveness, general physical self-worth, and potentially overall self-esteem.

Few studies have investigated the relationship between exercise-induced changes to the body and changes in self-perceptions, and the findings have been inconsistent suggesting that further research is required. Taylor and Fox (2005) reported that participants' changes in anthropometric measures were related to changes in physical self-perception in a primary care exercise referral program. However, Ransdell, Detling, Taylor, Reel, and Shultz (2004), did not find a relationship between changes in body mass and changes in physical self-perceptions in a mother and daughter sample following a 3-month fitness building exercise program. Ransdell et al. suggested that this finding might be due to the limited changes in the body mass of the participants. Use of additional anthropometric (e.g., body size) and body composition measures, which are more sensitive indexes of physical changes than body mass, may have provided greater insight into the relationship between physical changes and changes in self-perceptions. Alternatively Fox (2000) suggested that actual physical changes may be unnecessary, but instead the subjective feeling that one's body is improving through exercise may be sufficient to improve self-perceptions.

If the relationship between exercise and self-perceptions is mediated, at least partially, by changes in anthropometric measures and body composition then it may be expected that physical activity that targets specific body changes would lead to greater self-perception changes than alternative exercise, and no exercise. For example, there is some evidence to suggest that resistance exercise may be more effective than endurance exercise in improving body image, perhaps because it focuses on developing the muscle tone and strength of the body (Fox, 2000; Tucker \& Mortell, 1993; Williams \& Cash, 2001). By comparing the effects on self-perceptions of exercise that focuses on body changes with exercise that does not focus on body changes to the same extent, it may be possible to identify whether these body size, body mass and body composition changes are important in enhancing self-perceptions.

Brisk walking is a recommended mode of moderate intensity physical activity to achieve health and fitness benefits consistent with reduced cardiovascular risk (Murphy \& Hardman, 1998). Previous research has demonstrated that a program of moderate intensity walking $(\sim 65 \% \max$ 
HR) can result in improved self-esteem (Palmer, 1995), and body satisfaction (Brown et al., 1995), although limited research has considered the mechanisms for this change. Abdominal electrical muscle stimulation (EMS) devices have been marketed to increase strength, reduce size, and improve appearance of the abdominal area. Research on the effectiveness of EMS has produced inconsistent results (Parisi, Pigozzi, \& Prinzi, 1999; Porcari et al., 2002); however, a recent study suggested that EMS can lead to improvements in muscular strength and endurance of the abdominal region as well as participant's satisfaction with the abdominal area (Porcari et al., 2005). Dissatisfaction with one's abdomen can contribute to a negative body image (Garner, Olmstead, \& Polivy, 1983); therefore specific exercise, such as EMS, that targets improvements in this area may enhance body image and other self-perceptions. Thus it may be suggested that use of an abdominal EMS device in combination with brisk walking represents a form of physical activity that places greater focus on body mass, size, and muscle tone changes than brisk walking alone.

The aim of this randomized-controlled study was to compare the effects of an 8-week program of regular brisk walking, regular brisk walking with EMS, and no exercise on body changes, and hierarchical self-perception measures in sedentary adult women. The program duration was limited to 8 weeks to minimize attrition rates and because previous studies have demonstrated a change in anthropometric and body composition measures within this period (Murphy, Nevill, Biddle, Neville, \& Hardman, 2002). Although, it has been suggested that programs longer than 8 weeks are more beneficial on self-perceptions, changes have been evident at 8 weeks (Fox, 2000). In contrast to previous studies, which have typically included three sessions of activity per week (Fox, 2000), the activity program in this study was prescribed to adhere to current health recommendations (i.e., moderate intensity activity for $30 \mathrm{~min}$ on at least 5 days per week; Department of Health, 2004).

Four hypotheses were tested: (1) walking and walking + EMS will result in greater reduction in anthropometric measures and body composition than no walking; (2) walking and walking + EMS will result in greater improvements in self-perceptions than no walking; (3) the walking + EMS condition has greater focus on improvements in body size and muscle tone and will therefore result in greater changes in the anthropometric and body composition measures leading to superior increases in self-perceptions than the walking alone condition and no exercise; (4) exercise-induced improvements in self-perception will be mediated by improvements in anthropometric measures and body composition.

\section{Methods}

\section{Participants}

Participants were recruited through electronic mail to all female staff at a University (approx. $n=1500$ ) inviting interested sedentary women to volunteer to participate in a brisk walking program. Two hundred and three staff members responded and 74 submitted applications to participate in the study and were screened for general health status and physical activity levels. Exclusionary criteria included: being physically active (i.e., meeting the current health recommendations), age $>60$ years, cardiovascular, pulmonary or metabolic disease, smoking, 
pregnancy, Body Mass Index $(\mathrm{BMI})>40 \mathrm{~kg} \mathrm{~m}^{-2}$, and resting blood pressure $>159 / 99 \mathrm{~mm} \mathrm{Hg}$. Following health screening, 37 participants (mean age $=38.1 ; \mathrm{SD}=9.3$ ) were invited to attend an initial baseline testing and were subsequently individually randomly assigned to one of the three conditions. Written informed consent was obtained from all participants.

\section{Measures}

A number of anthropometric measures were used to monitor body size and body mass changes. Height $(\mathrm{m})$ and body mass $(\mathrm{kg})$ were recorded using a stadiometer (Seca model 770, Vogel \& Halke; Hamburg, Germany) and scales (Seca model 707 digital physicians scale; Vogel \& Halke, Hamburg, Germany), respectively. BMI was calculated by dividing body mass $(\mathrm{kg})$ by height $\left(\mathrm{m}^{2}\right)$. Body circumference measurements were made at the waist, umbilicus, hips, and mid-thigh. Waist measurements were made at the level of the trunk where the girth is minimal, (i.e. the location where there was a noticeable indentation of the trunk) and at the umbilicus. Hip girth was the horizontal circumference at the broadest part of the lower body, usually at the level of the trochanters (Jones, Hunt, Brown, \& Norgan, 1986). Mid-thigh measurements were made at the mid-point between the inguinal crease and the anterior patella. Body composition was assessed using bioelectrical impedance analysis to assess body fat percentage following recommended procedures (American Colleges of Sport Medicine, 2000).

Multi-dimensional self-perceptions were assessed at different levels of specificity (see Fig. 1). Specifically, a global measure of self-esteem was assessed using Rosenberg's (1965) Self-Esteem Scale, which includes 10 items (e.g., 'I feel that I have a number of good qualities'). The domain of physical self-worth and its associated sub-domains (sport competence, physical strength, physical condition, and body attractiveness) were all assessed using the Physical Self Perception Profile

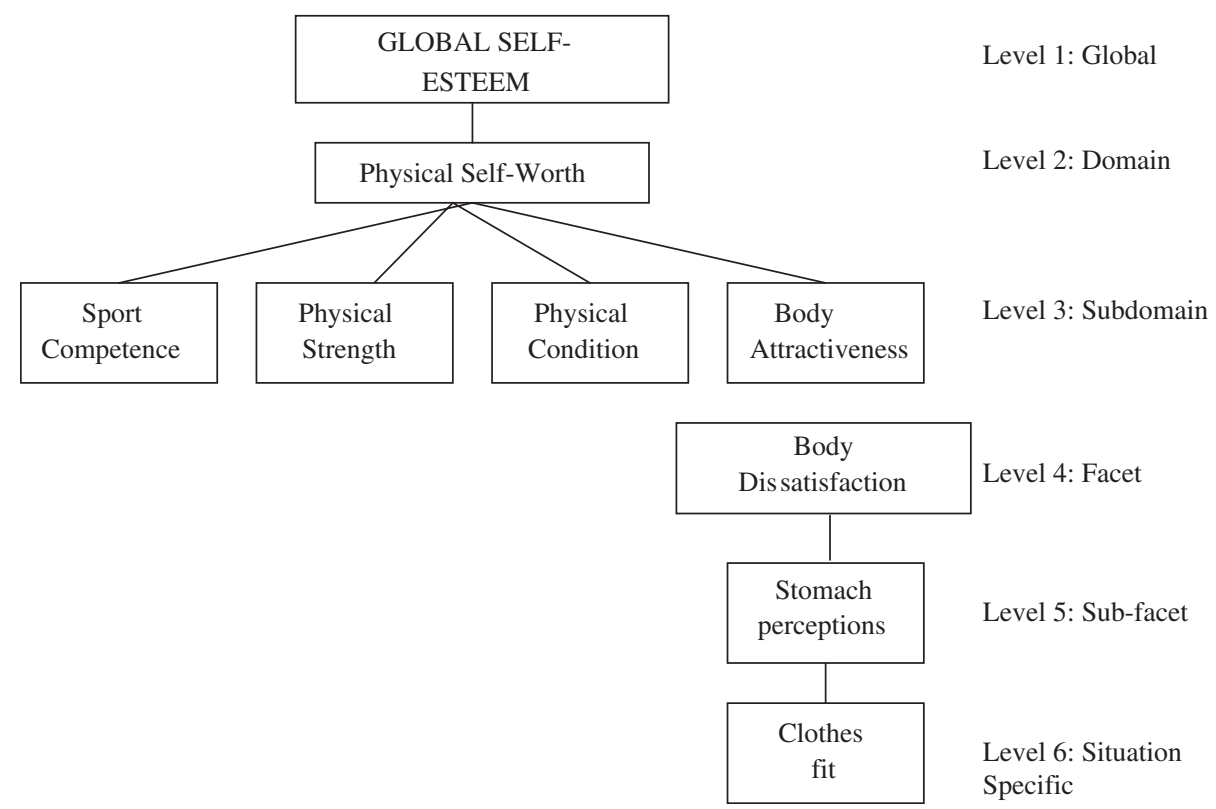

Fig. 1. Hierarchical self-perception measures in this study. 
(PSPP; Fox, 1990; Fox \& Corbin, 1989). The PSPP includes 30 items and participants are presented with two contrasting descriptions and are asked to determine which description is most like them and whether the description is "sort of true" or "really true" for them (e.g., "Some people tend to feel a little uneasy in fitness and exercise settings but others feel confident and at ease at all times in fitness and exercise settings'). More specific measures were also included to consider the facet, sub-facet and state levels of physical self-perception relevant to this study (Fox, 1998). The Body Dissatisfaction Scale (BDS) from the Eating Disorder Inventory (Garner et al., 1983) was used to measure body image. The BDS includes nine items (e.g., 'I think that my stomach is too big'), and was used to assess dissatisfaction with specific body parts that have been related to women's weight concerns. A questionnaire developed for this study that demonstrated good internal reliability (Cronbach alpha $=.92$ ) was used to assess perceptions of stomach appearance by responding to 12 adjective pairings on a 7-point scale (e.g., thin-fat). A one-item question was used to assess how a self-selected item of clothing felt on a 9-point scale from 'too tight' to 'too loose'. Feedback from participants indicated that this measure was appropriate and had face validity.

\section{Procedure}

The local University Ethics Committee granted ethical approval for the study. At baseline testing the participants completed the self-perception questionnaires, and anthropometric and body composition measures were taken, and physical fitness was assessed (fitness data not reported here). At the end of baseline testing session, the participants were individually randomly assigned to one of the three conditions by selecting a sealed envelope from a number of identical envelopes. The exercising conditions lasted 8 weeks and were designed to conform to current health recommendations for physical activity (Department of Health, 2004). Participants assigned to the walk group were instructed to walk briskly for 30 min on 5 days of each week. Participants assigned to the walking + EMS group were instructed to use abdominal EMS while walking briskly on 5 days of the week. All exercisers were given target heart rates and heart rate monitors in order to ensure their walking was at moderate intensity. During week 7, the researchers monitored the intensity of the walking to ensure that the selected intensity was indeed moderate. EMS is a low-voltage stimulation device worn around the waist that delivers a constant current output of $0-70 \mathrm{~mA}$ with a pulse duration of $200 \mu \mathrm{s}$ and a frequency of $40 \mathrm{~Hz}$. The EMS was set to a ramped protocol whereby the stimulation increased over $4 \mathrm{~s}$ up to the selected maximum intensity that was then administered for $10 \mathrm{~s}$ prior to decreasing again over a period of $4 \mathrm{~s}$. Participants were asked to choose the highest tolerable intensity and encouraged to progress the maximum intensity of the EMS each week. In week 1 participant chose levels 15-60 (equivalent to 10-42 mA) and by week 8 they had progressed to levels 62-99 (equivalent to 44-70 mA).

In order to assess program adherence participants were given a training diary to record their walks and note the day, time of day, and duration of the walk. Participants were also required to rate their perceived exertion during the walk on the Borg 15-grade scale (Borg, 1982). In addition, participants in the walking + EMS group were asked to record the level of muscle stimulation used. All participants were instructed regarding the importance of maintaining their usual activity and dietary habits throughout the study. 


\section{Analysis}

One-way ANOVAs were calculated to examine pre-intervention differences between the three groups. Repeated measure 2 (time) $\times 3$ (group) ANOVAs with follow-up one-way ANOVAs where appropriate were undertaken to examine the effect of each experimental condition on anthropometric and body composition measures and hierarchical self-perceptions. Estimates of effect sizes and power were calculated during the $2 \times 3$ ANOVAs. In order to investigate the influence of changes in anthropometric measures on self-perception changes, mediation analyses were conducted following the guidelines of Baron and Kenny (1986). Mediation occurs when the predictive association between a given predictor variable (e.g., exercise condition) and a criterion variable (e.g., self-perception) is reduced when taking into account a second predictor variable (e.g., body mass), which is predicted by the first predictor variable and itself predicts the criterion variable (Baron \& Kenny, 1986). Specifically, regression equations were calculated to determine the mediating effect of change in anthropometric measures on the predictive relationship between exercise condition and self-perceptions. Only those variables that changed significantly following the intervention were included. That is, the mediating influence of body mass, BMI, circumference of hip, umbilicus, waist, and thigh on exercise-related changes in body dissatisfaction, physical condition, stomach appearance, and clothes was investigated.

\section{Results}

Fig. 2 details the trial profile summarizing participant flow and numbers throughout the study. At the completion of the 8-week program, five participants had dropped out or were excluded due to ill health, substantial change in diet, and job pressures. All remaining participants $(n=32)$ were re-assessed on the self-perception, anthropometric, body composition, and fitness measures. Unfortunately, four participants failed to return the questionnaires and did not respond to followup requests, so were excluded from the final analysis. In some instances the participants failed to complete individual questionnaires (e.g., clothes fit), but were retained in the study to include the anthropometric data and data from the other questionnaires.

Despite randomization, differences between the three groups who completed the study did exist at baseline on the stomach appearance $\left(F_{(2,23)}=4, p=.03\right)$ and clothes fit $\left(F_{(2,20)}=4.5, p=.02\right)$ variables. Specifically, the control group reported significantly better clothes fit than walk group $(p=.02)$, and scored higher than the walking + EMS group on the stomach appearance questionnaire $(p=.03)$.

Overall adherence to the program was excellent with the walking group and walking + EMS completing $96.9 \%(\mathrm{SD}=4.9)$ and $95.3 \%(\mathrm{SD}=6.9)$ of the prescribed exercise. The intensity of exercise was assessed in week 7 and the percentage of maximum heart rate for each group was comparable (walking mean $=74.1 \%$; $\mathrm{SD}=5.0$; walking + EMS mean $=73.9 \%$; $\mathrm{SD}=4.7$ ). Rate of perceived exertion was also similar (walking mean $=12.3$; $\mathrm{SD}=1.0$; walking + EMS mean $=12.4 ; \mathrm{SD}=4.7$ ). Participants in the walking + EMS group self-selected a mean stimulation intensity of 60.8 (8.5) starting at a mean of 24.8 (5.8) in week 1 and progressing to a mean of $85.2(12.6)$ by week 8 . 


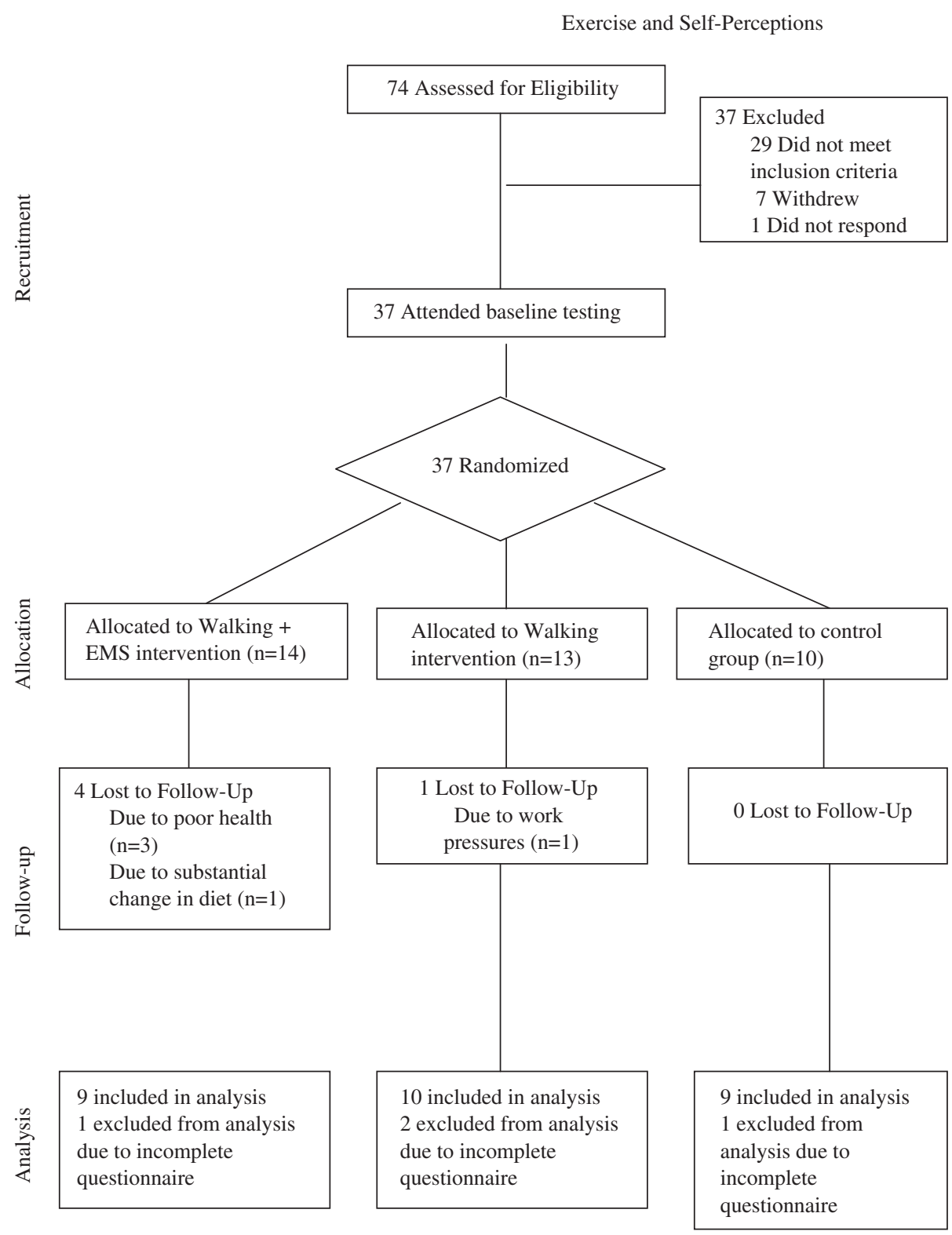

Fig. 2. Trial profile showing the progress of participants through the randomized trial.

\section{Anthropometric and body composition measures}

Table 1 illustrates pre- and post-intervention descriptive data for the anthropometric and body composition measures for each group. Results indicated a significant interaction effect between group and time for body mass $\left(F_{(2,26)}=4.07, p=.029, \eta_{\mathrm{p}}^{2}=.24\right.$, power $\left.=.67\right)$, BMI $\left(F_{(2,26)}=3.75, p=.036, \eta_{\mathrm{p}}^{2}=.24\right.$, power $\left.=.64\right)$, and circumferences of hip $\left(F_{(2,26)}=6.45\right.$, 
Table 1

Pre and post mean and standard deviation scores for each anthropometric measure for each group

\begin{tabular}{|c|c|c|c|c|c|c|}
\hline \multirow[t]{3}{*}{ Measure } & \multicolumn{6}{|c|}{ Experimental group } \\
\hline & \multicolumn{2}{|c|}{ Walk $(n=10)$} & \multicolumn{2}{|c|}{ Walk + EMS $(n=9)$} & \multicolumn{2}{|c|}{ Control $(n=9)$} \\
\hline & Pre & Post & Pre & Post & Pre & Post \\
\hline Body mass (kg) & $73.0(17.4)$ & $72.6(17.6)$ & $72.6(8.6)$ & $71.4(7.8)$ & $62.0(8.6)$ & $62.8(9.3)$ \\
\hline $\mathrm{BMI}\left(\mathrm{kg} \mathrm{m}^{2}\right)$ & $26.7(5.6)$ & $26.5(5.7)$ & $27.4(3.7)$ & $26.9(3.6)$ & $23.1(3.6)$ & $23.5(3.8)$ \\
\hline Hips $(\mathrm{cm})$ & $108.3(11.0)$ & $106.2(9.6)$ & $107.6(7.7)$ & $105.8(7.0)$ & $97.4(6.0)$ & $98.7(6.8)$ \\
\hline Waist narrowest $(\mathrm{cm})$ & $81.6(13.6)$ & $80.9(14.3)$ & $83.0(6.7)$ & $80.9(6.7)$ & $73.7(9.8)$ & $74.7(9.6)$ \\
\hline Thigh $(\mathrm{cm})$ & $53.2(5.3)$ & $51.4(4.9)$ & $54.1(4.8)$ & $52.8(4.8)$ & $48.9(4.0)$ & $49.5(4.1)$ \\
\hline Waist umbilicus (cm) & $95.0(16.1)$ & $95.4(17.5)$ & $94.6(8.8)$ & $92.8(7.8)$ & $83.9(11.1)$ & $87.0(10.8)$ \\
\hline Fat $(\%)$ & $35.6(6.5)$ & $35.5(6.2)$ & $36.1(6.3)$ & $35.7(6.5)$ & $30.4(7.1)$ & $30.7(6.7)$ \\
\hline
\end{tabular}

$p=.005, \eta_{\mathrm{p}}^{2}=.33$, power $\left.=.87\right)$, umbilicus $\left(F_{(2,26)}=7.90, p=.002, \eta_{\mathrm{p}}^{2}=.38\right.$, power $\left.=.93\right)$, waist $\left(F_{(2,26)}=3.52, p=.044, \eta_{\mathrm{p}}^{2}=.21\right.$, power $\left.=.60\right)$, and thigh $\left(F_{(2,25)}=4.75, p=.018, \eta_{\mathrm{p}}^{2}=.28\right.$, power $=.74)$. Effect sizes indicated that group and time interaction accounted for at least $21 \%$ of variance in each variable, and analyses indicated the study had sufficient power (Cohen, 1992). Follow-up planned comparisons of the change between pre- and post-condition for each group on each measure were undertaken using one-way ANOVAs and appropriate post-hoc analyses. Both walking + EMS group and the walking group had significantly greater changes in hip circumference (walking + EMS, $p=.011$; walking, $p=.011$ ) than the control group. Additionally, the walking + EMS group had significantly greater change in body mass $(p=.023)$, BMI $(p=.029)$, and waist $(p=.035)$ than the control group. The walking group had significantly greater change in thigh circumference $(p=.006)$ than the control group. Interestingly, the increase in the umbilicus circumference of the control group was significantly greater $(p=.001)$ than the decrease in umbilicus for the walking + EMS group. There were no significant differences between the groups on changes in body fat percentage.

\section{Self-perceptions}

Table 2 illustrates pre- and post-intervention descriptive data for self-perception measures for each group. There was a significant interaction effect for group and time for body dissatisfaction $\left(F_{(2,23)}=4.62, p=.021, \eta_{\mathrm{p}}^{2}=.29\right.$, power $\left.=.72\right)$, physical condition $\left(F_{(2,25)}=3.28, p=.05\right.$, $\eta_{\mathrm{p}}^{2}=.21$, power $\left.=.57\right)$, stomach appearance $\left(F_{(2,23)}=9.39, p=.001, \eta_{\mathrm{p}}^{2}=.45\right.$, power $\left.=.96\right)$, and clothes fit $\left(F_{(2,16)}=8.65, p=.003, \eta_{\mathrm{p}}^{2}=.52\right.$, power $\left.=.93\right)$. Effect sizes indicated that group and time interaction accounted for at least $21 \%$ of variance in each variable, and analysis indicated that the study had sufficient power. Follow-up planned one-way ANOVAs with appropriate post-hoc analyses indicated that the walking + EMS group $(p=.01)$ and the walk group $(p=.006)$ had significantly greater changes in clothes fit than the control group. Additionally, there was a significant difference between the walking + EMS group and the control group on decreases in body dissatisfaction $(p=.024)$, and improvements on stomach appearance 
Table 2

Pre and post mean and standard deviation scores for each self-perception measure for each group

\begin{tabular}{|c|c|c|c|c|c|c|}
\hline \multirow[t]{3}{*}{ Measure } & \multicolumn{6}{|c|}{ Experimental group } \\
\hline & \multicolumn{2}{|c|}{ Walk $(n=10)$} & \multicolumn{2}{|c|}{ Walk + EMS $(n=9)$} & \multicolumn{2}{|c|}{ Control $(n=9)$} \\
\hline & Pre & Post & Pre & Post & Pre & Post \\
\hline Self-esteem $(\max .=40)$ & $30.6(5.0)$ & $31.7(5.0)$ & $29.1(5.3)$ & $29.4(4.1)$ & $31.0(4.2)$ & $33.6(4.0)$ \\
\hline Physical self-worth $(\max .=24)$ & $11.4(2.4)$ & $12.0(1.6)$ & $9.5(1.8)$ & $12.0(1.7)$ & $13.6(4.7)$ & $13.7(4.3)$ \\
\hline Sport $(\max .=24)$ & $10.8(3.2)$ & $11.4(3.0)$ & $9.2(4.0)$ & $9.4(3.5)$ & $10.3(3.0)$ & $11.6(4.4)$ \\
\hline Strength $(\max .=24)$ & $12.4(1.3)$ & $12.6(2.4)$ & $10.3(3.4)$ & $11.2(2.9)$ & $11.8(3.3)$ & $11.7(3.1)$ \\
\hline Conditioning $(\max .=24)$ & $9.7(2.1)$ & $11.7(1.3)$ & $9.2(2.8)$ & $10.4(2.2)$ & $10.1(1.8)$ & $10.4(2.2)$ \\
\hline Body attractiveness $(\max .=24)$ & $10.4(3.3)$ & $11.3(3.1)$ & $10.0(3.4)$ & $12.0(2.0)$ & $14.0(5.1)$ & $13.8(5.3)$ \\
\hline Body dissatisfaction $(\max .=27)$ & $15.8(6.6)$ & $13.2(6.4)$ & $17.2(5.8)$ & $14.0(7.3)$ & $9.4(8.6)$ & $11.3(7.3)$ \\
\hline Stomach appearance $(\max .=74)$ & $31.2(12.1)$ & $37.3(11.8)$ & $25.9(7.0)$ & $39.7(10.1)$ & $42.0(14.6)$ & $36.9(13.1)$ \\
\hline Clothes fit $(\max .=9)$ & $2.6(1.5)$ & $4.8(1.9)$ & $2.9(4.1)$ & $5.5(.5)$ & $4.3(.5)$ & $3.8(1.5)$ \\
\hline
\end{tabular}

( $p=.001)$ from pre- to post-intervention. The walking group had significantly greater change in physical condition than the control group $(p=.05)$.

\section{Mediation effect of changes in anthropometric measures on exercise-induced self-perception changes}

A mediating influence of changes in anthropometric measures on exercise-induced selfperceptions was evident only for body mass, BMI, and thigh circumference on the clothes fit perception. The results from these 3-step regression analyses are presented in Table 3 . As is evident, in each analysis at Step 3 the mediating variables body mass, BMI, and circumference of thigh had a significantly greater influence on the change in perception of clothes fit than the predictive variable of exercise condition.

\section{Discussion}

Exercise has been associated with improvements in self-perceptions, however there has been limited research examining the influence of exercise that conforms with current health recommendations on self-perceptions, the influence of exercise on the hierarchy of selfperceptions, and there is currently a limited understanding of the mechanisms that influence exercise-self-perception relationship (Fox, 2000). The purpose of this study was to investigate and compare the effects of an 8-week program of brisk walking, brisk walking plus abdominal EMS, and no exercise on anthropometric and body composition measures and a hierarchy of selfperceptions, and consider the role of body changes in the exercise-self-perception relationship.

Supporting the first hypothesis and previous research, both exercising groups exhibited significant changes in anthropometric measures compared to the control group (Murphy \& Hardman, 1998). The walking-only group had significantly greater reductions in hip circumference and thigh circumference than the control group. However, the walking + EMS 
Table 3

Series of regression analyses to test the mediation effect of physical changes on exercise-induced changes in the selfperception measure of clothes fit

\begin{tabular}{llccc}
\hline Step & & $B$ & $S E B$ & $\beta$ \\
\hline 1 & Condition regressed on clothes fit & 2.1 & .6 & $.63^{* *}$ \\
2 & Body mass regressed on condition & -.23 & .05 & $-.48^{* *}$ \\
3 & Condition & .98 & .74 & .30 \\
& Body mass regressed on clothes fit & -.71 & .31 & $-.51^{* *}$ \\
1 & Condition regressed on clothes fit & 2.1 & .6 & $.63^{* *}$ \\
2 & BMI regressed on condition & -.60 & .22 & $-.47^{* *}$ \\
3 & Condition & .95 & .70 & .29 \\
& BMI regressed on clothes fit & -2.01 & .80 & $-.54^{* *}$ \\
1 & Condition regressed on clothes fit & 2.1 & .6 & $.63^{* *}$ \\
2 & Thigh circumference regressed on condition & -.17 & .08 & $-.40^{*}$ \\
3 & Condition & .71 & .65 & .22 \\
& Thigh circumference regressed on clothes fit & -.88 & .27 & $-.65^{* *}$ \\
\hline
\end{tabular}

Note: $* * p<.01 ; * p<.05$.

group exhibited greater changes than the walking-only group compared with the control group. Specifically, significant decreases were evident for hip circumference, body mass, BMI, and waist circumference compared with the control group. Further, although the mean umbilicus circumference for the walking + EMS group decreased this was not significantly different to the walking group, but the increase of the umbilicus circumference of the control group was significantly greater than this decrease. This finding may indicate that walking + EMS could serve a protective function preventing an increase in umbilicus circumference. It appears that an 8-week program of walking + EMS has a greater effect on anthropometric measures than walking alone, providing support for the contention that this condition had greater focus on body changes than walking alone. This finding adds support to previous EMS research that has shown significant decreases in body size compared with a control group (e.g., Porcari et al., 2005).

Compared with the non-active control group, significant improvements in self-perceptions were evident for both the exercising groups. This finding supports previous research that suggests exercise enhances self-perceptions (e.g., Asçi, 2002, 2003; Taylor \& Fox, 2005) and is contrary to other studies that have found that exercise does not improve self-perceptions (e.g., Asçi et al., 1998; Caruso \& Gill, 1992). Improvements in self-perception were particularly evident in the constructs at the lower end of the self-perception hierarchy (see Fig. 1). Specifically, both the walk and walking + EMS groups had significantly greater changes in the state measure perception of clothes fit than the control group. The walking + EMS group also experienced significantly greater improvements than the control group at the facet (body dissatisfaction) and sub-facet (perceptions of stomach appearance) levels. Significant changes at the higher sub-domain levels of physical self-worth were not evident, with the exception of the walking group that had a significantly greater improvement on the sub-domain physical condition than the control group. Changes in the physical strength and sport competence subscales were minimal, and probably 
reflect the nature of the interventions that were neither strength nor sport focused. There were no significant changes in the higher-order domain level construct of physical self-worth, although non-significant improvements in the higher-order self-perception of physical self-worth were evident for both the experimental groups but not the control group, with greater improvements exhibited by the walking + EMS group. There were also no significant changes at the global level of self-esteem following the program. These findings are consistent with current theoretical understanding that suggests the relationship between exercise and self-perceptions appears to be stronger and more consistent for the lower levels of the self-perception hierarchy (Fox, 2000), and support previous research that has found these self-perceptions more susceptible to change following exercise (Alfermann \& Stoll, 2000; Brown et al., 1995). Self-esteem is a stable construct made up of several life domains and is less susceptible to change than the lower levels of the selfperception hierarchy, and as such changes following an 8-week program would not be expected (Sonstroem \& Morgan, 1989; Sonstroem et al., 1994). A longer experimental period may have resulted in greater changes in the more stable self-perception constructs. Further research adopting a time-series design could be valuable in investigating the onset of exercise-induced changes at different levels of the self-perception hierarchy.

It is difficult to explain why the significant improvement on the physical condition subscale for the walking-only group was not also evident for the walking + EMS group, who completed and adhered to the same walking program as the walkers. It may be that for the walking group selfperceptions related to physical conditioning (i.e., level of physical condition, stamina and fitness, and ability to maintain exercise and confidence in exercise and fitness settings; Fox, 1990) were more salient during the program than for the walking + EMS group who may have been more focused on body size changes due to their use of the EMS belt. It is of interest that compared with the control group, the walking + EMS group improved significantly on the body dissatisfaction subscale, but did not improve on the similar measure of the body attractiveness subscale of the PSPP. However, these measures do differ on their level specificity, as the body dissatisfaction measure focuses on specific body areas whereas the body attractiveness subscale includes assessment of a perception of one's general body attractiveness. This finding provides further support for the suggestion that self-perception constructs at the lower end of the hierarchy are more malleable to change (Fox, 2000). Future studies should include measures of self-perception at each of the levels in order to accurately monitor exercise-induced changes. A limitation of this study is that the state-specific measure of clothes fit was only a one-item question. Although participants reported that this measure had face validity, future research should develop a more comprehensive measure.

It should be acknowledged that despite randomization at baseline the control group had significantly more positive perceptions relating to stomach appearance than the walking + EMS group, and clothes fit than the walking group. This unfortunate non-equivalence at baseline makes interpretation more complex, and could indicate that the data represent a regression towards the mean or that there was a stronger likelihood of change in the exercise groups on these variables.

It was hypothesized that if exercise-induced changes in self-perceptions are mediated by improvements in anthropometric measures and body composition then the exercise activity of walking + EMS, which has more focus on these attributes, would result in greater changes in selfperception. Although both exercising groups demonstrated significant improvements in the 
anthropometric measures, the changes for the walk-only group were not as marked as for the walking + EMS group. Further, as noted above, greater changes in self-perception were evident for the walking + EMS group than for the walk-only group. It is suggested that the greater changes in self-perceptions reported by the walking + EMS group compared with the walk group are as a result of the greater changes in anthropometric measures that the walking + EMS group experienced. These findings provide some support for the hypothesis that exercise-induced improvements in state and sub-domain self-perceptions, such as clothes fit, stomach appearance, and body dissatisfaction, are mediated by changes in body size and body mass. This finding supports previous research that has found activity with greater focus on body shape changes that led to greater improvements in body image (e.g., Tucker \& Mortell, 1993).

However, these findings are not supported by the results of the mediation analysis examining the independent influence of changes in anthropometric measures on changes in self-perceptions, as limited significant effects were evident. From this study, it appears that the physical changes of body mass, BMI, and thigh circumference mediate the influence of exercise only on the state measure of perceptions of clothes fit. A mediation effect of physical changes was not evident for self-perceptions at higher levels of the hierarchy. These results could indicate that objective changes in anthropometric measures are not strongly related to changes in self-perception, and support the findings of Ransdell et al. (2004). It may be proposed that the relationship between objective body changes and self-perceptions is not strong because only small changes in body size are necessary to achieve large improvements in self-perceptions. Indeed, Fox (2000) has suggested previously that the extent of objective body changes itself is not important, but instead the subjective feeling that one's body is improving through exercise could lead to improved selfperceptions. Alternatively, it could be that an earlier mediation effect of physical changes is only evident on state measures of self-perception and over time these may become more influential on higher-level self-perceptions. For example, Taylor and Fox (2005) found that 16 weeks after the initiation of exercise there was a limited relationship between anthropometric measures and selfperception changes, but a follow-up study at 37 weeks revealed greater evidence of a relationship. Further research is required to investigate the mediation effect of physical changes on exerciseinduced self-perception changes over a longer period of time.

On the other hand, the improvements in self-perceptions evident from walking and the greater improvements resulting from walking + EMS may be due to another mechanism not considered in this study, such as increased personal control or an undetermined psychophysiological mechanism (Fox, 2000). Alternatively, the improvements in self-perception following exercise may not be a result of the exercise per se, but are due to an expectancy or attentional effect (Alfermann \& Stoll, 2000; Desharnais et al., 1993). This explanation is difficult to discount, as a limitation of this study is that it did not include an attentional placebo group. Future research should include a placebo group in order to further investigate the influence of expectancy effect on improvements in selfperception.

\section{Conclusions}

The findings of this study indicate that an 8-week program of exercise conforming to current health recommendations (Department of Health, 2004) results in greater improvements in 
anthropometric measures and self-perceptions than no exercise. Following an 8-week exercise program changes in self-perception are more evident at the lower end of the self-perception hierarchy, and less so at the more stable global, domain and sub-domain levels. Walking + EMS is more beneficial in reducing anthropometric measures, and improving self-perceptions than walking alone. This finding could indicate that exercise-induced changes in body size and body mass are influential in facilitating exercise-induced changes in some self-perception constructs. However, additional research is required to examine this mediating effect further. Future research should also aim to address the limitations of this study by including a placebo attention control group, ensuring equal groups following randomization, developing a more comprehensive measure of perceptions of clothes fit, and increasing sample size.

\section{Acknowledgments}

The authors would like to acknowledge the support of Biomedical Research Ltd. (Galway, Ireland) who provided sponsorship for the research and supplied the abdominal muscle stimulation devices.

\section{References}

Alfermann, D., \& Stoll, O. (2000). Effects of physical exercise on self-concept and well-being. International Journal of Sport Psychology, 30, 47-65.

American College of Sport Medicine. (2000). Guidelines for exercise testing and prescription (6th ed). Baltimore: Williams \& Wilkins.

Asçi, F. H. (2002). The effects of step dance on physical self-perception of female and male university students. International Journal of Sport Psychology, 33, 431-443.

Asçi, F. H. (2003). The effects of physical fitness training on trait anxiety and physical self-concept of female university students. Psychology of Sport and Exercise, 4, 255-264.

Asçi, F. H., Kin, A., \& Kosar, N. (1998). Effects of participation in an 8 week aerobic dance and step aerobics program on physical self-perception and body image satisfaction. International Journal of Sport Psychology, 29, 366-375.

Bailey, S. M., Goldbergh, J. P., Swap, W. C., Chomitz, V. R., \& Houser, R. F. (1990). Relationships between body dissatisfaction and physical measurement. International Journal of Eating Disorders, 9, 457-461.

Bane, S., \& McAuley, E. (1998). Body image and exercise. In J. Duda (Ed.), Advances in sport and exercise measurement (pp. 311-324). Morgantown, WV: Fitness Information Technology.

Baron, R. M., \& Kenny, D. A. (1986). The moderator-mediator variable distinction in social psychological research: Conceptual, strategic and statistical consideration. Journal of Personality and Social Psychology, 51, 1173-1182.

Biddle, S. J. H., \& Mutrie, N. (2001). Psychology of physical activity. London: Routledge.

Borg, G. A. V. (1982). Psychophysical bases of perceived exertion. Medicine and Science in Sports and Exercise, 14, 377-381.

Brown, D. R., Wang, Y., Ward, A., Ebbeling, C. B., Fortlage, L., Puleo, E., et al. (1995). Chronic psychological effects of exercise and exercise plus cognitive strategies. Medicine and Science in Sports and Exercise, 27, 765-775.

Caruso, C. M., \& Gill, D. L. (1992). Strengthening physical self-perceptions through exercise. Journal of Sports Medicine and Physical Fitness, 32, 416-427.

Cohen, J. (1992). A power primer. Psychological Bulletin, 112, 155-159.

Davis, C. (1997). Body image, exercise, and eating behaviors. In K. R. Fox (Ed.), The physical self: From motivation to well-being (pp. 143-174). Champaign, IL: Human Kinetics. 
Department of Health. (2004). At least five a week: Evidence of the impact of physical activity and its relationship to health. A report from the Chief Medical Officer. London: Department of Health.

Desharnais, R., Jobin, J., Cote, C., Levesque, L., \& Godin, G. (1993). Aerobic-exercise and the placebo effect-A controlled study. Psychosomatic Medicine, 55(2), 149-154.

Fox, K. R. (1990). The physical self perception profile manual. DeKalb, IL: Office for Health Promotion, Northern Illinois University.

Fox, K. R. (1998). Advances in the measurement of the physical self. In J. L. Duda (Ed.), Advances in sport and exercise psychology measurement (pp. 295-310). Fitness Information Technology.

Fox, K. R. (2000). The effects of exercise on self-perceptions and self-esteem. In S. J. H., Biddle, K. R. Fox, \& S. H. Boutcher (Eds.), Physical activity and psychological well-being (pp. 88-117). London: Routledge.

Fox, K. R., \& Corbin, C. B. (1989). The physical self-perception profile: Development and preliminary validation. Journal of Sport and Exercise Psychology, 11, 408-430.

Garner, D. M., Olmstead, M. P., \& Polivy, J. (1983). Development and validation of a multidimensional eating disorder inventory for anorexia nervosa and bulimia. International Journal of Eating Disorders, 2, 15-34.

Jones, P. R. M., Hunt, M. J., Brown, T. P., \& Norgan, N. G. (1986). Waist-hip circumference ratio and its relation to age and overweight in British men. Human Nutrition: Clinical Nutrition, 40C, 239-247.

McAuley, E., Blissmer, B., Katula, J., Duncan, T. E., \& Mihalko, S. L. (2000). Physical activity, self-esteem and selfefficacy relationships in older adults: A randomized controlled trial. Annals of Behavioral Medicine, 22, 131-139.

Murphy, M. H., \& Hardman, A. E. (1998). Training effects of short and long bouts of brisk walking in sedentary women. Medicine and Science in Sports and Exercise, 30, 152-157.

Murphy, M. H., Nevill, A. M., Biddle, S. J. H., Neville, C., \& Hardman, A. E. (2002). Accumulating brisk walking for fitness, cardiovascular risk, and psychological health. Medicine and Science in Sports and Exercise, 34(9), 1468-1474.

Palmer, L. K. (1995). Effects of a walking program on attributional style, depression, and self-esteem in women. Perceptual and Motor Skills, 81, 891-898.

Parisi, P., Pigozzi, F., \& Prinzi, G. (1999). Effects of neuromuscular electrical stimulation on static and dynamic abdominal strength and endurance in healthy males. In Proceedings of fourth annual congress of the European college of sports science.

Porcari, J. P., McLean, K. P., Foster, C., Kernozek, T., Crenshaw, B., \& Swenson, C. (2002). Effects of electrical muscle stimulation on body composition, muscle strength, and physical appearance. Journal of Strength and Conditioning Research, 16, 165-172.

Porcari, J. P., Miller, J., Cornwell, K., Foster, C., Gibson, M., McLean, K., et al. (2005). The effects of neuromuscular electrical stimulation training on abdominal strength, endurance, and selected anthropometric measures. Journal of Sports Science and Medicine, 4, 66-75.

Ransdell, L. B., Detling, N., Taylor, A., Reel, J., \& Shultz, B. (2004). Daughters and Mothers Exercising Together (DAMET): Effects of home- and university-based programs on physical self-perception in mothers and daughters. Women and Health, 39, 63-81.

Rosenberg, M. (1965). Society and the adolescent self-image. Princeton, NJ: Princeton University Press.

Sonstroem, R. J. (1998). Physical self-concept: Assessment and external validity. Exercise and Sport Sciences Reviews, $28,133-164$.

Sonstroem, R. J., Harlow, L. L., \& Josephs, L. (1994). Exercise and self-esteem: Validity of model expansion and exercise associations. Journal of Sport and Exercise Psychology, 16, $29-42$.

Sonstroem, R. J., \& Morgan, W. P. (1989). Exercise and self-esteem: Rational and model. Medicine and Science in Sports and Exercise, 21, 329-337.

Taylor, A. H., \& Fox, K. R. (2005). Effectiveness of a primary care exercise referral intervention for changing physical self-perceptions over 9 months. Health Psychology, 24, 11-21.

Tucker, L. A., \& Mortell, R. (1993). Comparison of the effects of walking and weight training programs on body image in middle-aged women: An experimental study. American Journal of Health Promotion, 8, 34-42.

Williams, P. A., \& Cash, T. F. (2001). Effects of a circuit weight training program on the body images of college students. International Journal of Eating Disorders, 30, 75-82. 